\title{
DIAMETERS OF COMMUTING GRAPHS OF MATRICES OVER SEMIRINGS
}

\author{
DAVID DOLŽAN, DAMJANA KOKOL BUKOVŠEK, POLONA OBLAK
}

\begin{abstract}
We calculate the diameters of commuting graphs of matrices over the binary Boolean semiring, the tropical semiring and an arbitrary nonentire commutative semiring. We also find the lower bound for the diameter of the commuting graph of the semiring of matrices over an arbitrary commutative entire antinegative semiring.
\end{abstract}

\section{INTRODUCTION}

Definition. A semiring is a set $S$ equipped with binary operations + and $\cdot$ such that $(S,+)$ is a commutative monoid with identity element 0 , and $(S, \cdot)$ is a monoid with identity element 1 . In addition, operations + and $\cdot$ are connected by distributivity and 0 annihilates $S$. A semiring is commutative if $a b=b a$ for all $a, b \in S$.

A semiring $S$ is called antinegative, if $a+b=0$ implies that $a=b=0$. Antinegative semirings are also called antirings (or zero-sum-free semirings). A semiring is entire (or zero-divisor-free) if $a b=0$ implies that $a=0$ or $b=0$. A semiring is a division semiring if all nonzero elements have multiplicative inverses.

The simplest example of an antinegative semiring is the binary Boolean semiring, the set $\{0,1\}$ with $1+1=1 \cdot 1=1$. We will denote the binary Boolean semiring by $\mathcal{B}$.

Moreover, the set of nonnegative integers (or reals) with the usual operations of addition and multiplication, is a commutative entire antinegative semiring, and all distributive lattices are antinegative semirings.

On the set $\mathbb{R} \cup\{-\infty\}$, we define operations $a \oplus b=\max \{a, b\}$ and $a \odot b=a+b$, where $-\infty+a=a+(-\infty)=-\infty$. It is easy to verify that $(\mathbb{R} \cup\{-\infty\}, \oplus, \odot)$ is a semiring. It is denoted by $\mathbb{T}$ and called the tropical semiring, sometimes also the max-plus semiring. Tropical semiring is a commutative entire antinegative division semiring and it is closely related to the max algebra, i.e. the semiring of nonnegative reals $\mathbb{R}_{+}$, where the addition is the same as in the tropical semiring and the multiplication is the ordinary multiplication on reals. Recently, commuting matrices in max algebra were studied in [10].

Over past decades, the tropical semirings and other tropical structures were widely investigated. Let us mention only a few pioneering works [4, 5, 13, that connect the theory of matrices over classical and tropical worlds.

We denote by $\mathcal{M}_{n}(S)$ the semiring of all $n \times n$ matrices over a semiring $S$ and by $G L_{n}(S)$ the group of all invertible matrices in $\mathcal{M}_{n}(S)$. By $I_{n} \in \mathcal{M}_{n}(S)$, we denote the

Date: November 6, 2018.

2010 Mathematics Subject Classification. 15A27, 16Y60, 05C50, 05C12.

Key words and phrases. semiring, Boolean semiring, tropical semiring, commuting graph, diameter. 
identity matrix and by $0_{n} \in \mathcal{M}_{n}(S)$ the zero matrix. The matrix with the only nonzero entry 1 in the $i$-th row and the $j$-th column will be denoted by $E_{i, j}$.

If $X$ is a subset of $S$, let

$$
C_{S}(X)=\{s \in S ; s x=x s \text { for all } x \in X\}
$$

denote the centralizer of $X$ in $S$. For $x \in S$ we also define $C_{S}(x)=C_{S}(\{x\})$.

For any subset $T$ of a semiring $S$, we denote by $\Gamma(T)$ the commuting graph of $T$. The vertex set $V(\Gamma(T))$ of $\Gamma(T)$ is the set of elements in $T \backslash C_{S}(T)$. An unorderded pair of vertices $x-y$ is an edge of $\Gamma(T)$ if $x \neq y$ and $x y=y x$.

The sequence of edges $x_{0}-x_{1}, x_{1}-x_{2}, \ldots, x_{k-1}-x_{k}$ is called a path of length $k$ and is denoted by $x_{0}-x_{1}-\ldots-x_{k}$. The distance between two vertices is the length of the shortest path between them. The diameter of the graph is the longest distance between any two vertices of the graph $\Gamma$ and will be denoted by $\operatorname{diam}(\Gamma)$.

For example, the commuting graph of the set of all $2 \times 2$ nilpotent matrices over an entire antinegative semiring $S$, is a disconnected graph with two components (corresponding to the strictly upper, and strictly lower triangular matrices), where both components are isomorphic to a complete graph on $|S|-1$ vertices.

Recently, the commuting graphs of matrix rings and semirings $([1,2,13,6,7,6,11])$ and commuting graphs of various algebraic structures ([9, 12]) have been studied. They give an illustrative way of describing centralizers of elements. It was proved in [2, Cor. 7] that the diameter of the commuting graph of the full matrix ring over an algebraically closed

field is equal to 4. For rings and fields that are not algebraically closed, the commuting graph of the full matrix ring might not be connected at all, or if it is connected, its diameter can be larger than 4. (See e.g. [6, Ex. 2. 15], where it has been proven that $5 \leq \operatorname{diam}\left(\Gamma\left(\mathcal{M}_{9}\left(\mathbb{Z}_{2}\right)\right)\right)<\infty$. $)$

In this paper, we continue with the investigation of the diameters of commuting graphs of full matrix semirings. In [7, Prop. 10] it has been shown that the diameter of the commuting graph of the full matrix semiring over $\mathcal{B}$ is bounded between 3 and 4 . In the second section, we prove that it is equal to 4 . This implies that the diameter of the commuting graph of $\mathcal{M}_{n}(S)$ is at least 4 for every commutative entire antinegative semiring $S$. Using this, we prove in Theorem 3.2 that for the tropical semiring $\mathbb{T}$ the diameter of $\Gamma\left(\mathcal{M}_{n}(\mathbb{T})\right)$ is equal to 4 . In Section 4 , we prove that $\operatorname{diam}\left(\Gamma\left(\mathcal{M}_{n}(S)\right)\right)=3$ for every nonentire commutative semiring $S$ and all $n \geq 2$, thus generalizing [8, Thm. 1.1], where a similar result has been proven for $\mathcal{M}_{n}\left(\mathbb{Z}_{m}\right)$, where $m$ is not a prime number.

\section{The commuting graph of the Full matrix Semiring OVer $\mathcal{B}$}

In this section, we prove that the diameter of the commuting graph of the full $n \times n$ matrix semiring over Boolean semiring $\mathcal{B}$ is equal to 4 for all $n \geq 3$.

We start with a general lemma, which can be easily proved by a straightforward calculation. Denote by $J_{n}$ the nilpotent $n \times n$ matrix $E_{1,2}+E_{2,3}+\ldots+E_{n-1, n}$. 
Lemma 2.1. If $S$ is a semiring and $n \geq 2$, then the centralizer of $J_{n}$ is equal to $C_{\mathcal{M}_{n}(S)}\left(J_{n}\right)=\left\{a_{0} I_{n}+a_{1} J_{n}+a_{2} J_{n}^{2}+\ldots+a_{n-1} J_{n}^{n-1} ; a_{i} \in S\right\}$, and the centralizer of $J_{n}^{T}$ is equal to $C_{\mathcal{M}_{n}(S)}\left(J_{n}^{T}\right)=\left\{b_{0} I_{n}+b_{1} J_{n}^{T}+b_{2}\left(J_{n}^{T}\right)^{2}+\ldots+b_{n-1}\left(J_{n}^{T}\right)^{n-1} ; b_{i} \in S\right\}$.

The following Theorem is the main result of this section.

Theorem 2.2. If $\mathcal{B}$ is a binary Boolean semiring, then $\operatorname{diam}\left(\Gamma\left(\mathcal{M}_{2}(\mathcal{B})\right)\right)=\infty$ and

$$
\operatorname{diam}\left(\Gamma\left(\mathcal{M}_{n}(\mathcal{B})\right)\right)=4
$$

for $n \geq 3$.

Proof. It was proved in [7, Prop. 10] that $\operatorname{diam}\left(\Gamma\left(\mathcal{M}_{2}(\mathcal{B})\right)\right)=\infty$. Let $n \geq 3$ and let $E \in$ $\mathcal{M}_{n}(\mathcal{B})$ be a matrix of all ones. It was also proved in [7, Prop. 10] that the distance between any matrix $A \in \mathcal{M}_{n}(\mathcal{B})$ and matrix $E$ is at most 2. It follows that $\operatorname{diam}\left(\Gamma\left(\mathcal{M}_{n}(\mathcal{B})\right)\right) \leq 4$ for $n \geq 3$. To prove the equality, we have to find two matrices with the distance between them at least 4 .

Let $n=3$ and let

$$
A=\left[\begin{array}{lll}
0 & 0 & 1 \\
0 & 0 & 0 \\
1 & 1 & 0
\end{array}\right], \quad B=\left[\begin{array}{lll}
1 & 0 & 0 \\
0 & 0 & 1 \\
0 & 0 & 0
\end{array}\right]
$$

A straight-forward calculation shows that the set of all matrices with distance 1 to matrix $A$ is

$$
\mathbb{A}=\left\{\left[\begin{array}{lll}
1 & 0 & 1 \\
0 & 1 & 0 \\
1 & 1 & 1
\end{array}\right],\left[\begin{array}{lll}
1 & 1 & 0 \\
0 & 0 & 0 \\
0 & 0 & 1
\end{array}\right],\left[\begin{array}{lll}
1 & 1 & 0 \\
0 & 1 & 0 \\
0 & 0 & 1
\end{array}\right],\left[\begin{array}{lll}
1 & 1 & 1 \\
0 & 0 & 0 \\
1 & 1 & 1
\end{array}\right],\left[\begin{array}{lll}
1 & 1 & 1 \\
0 & 1 & 0 \\
1 & 1 & 1
\end{array}\right]\right\}
$$

and the set of all matrices with distance 1 to matrix $B$ is

$$
\mathbb{B}=\left\{\left[\begin{array}{lll}
1 & 0 & 0 \\
0 & 0 & 0 \\
0 & 0 & 0
\end{array}\right],\left[\begin{array}{lll}
1 & 0 & 0 \\
0 & 1 & 1 \\
0 & 0 & 1
\end{array}\right],\left[\begin{array}{lll}
0 & 0 & 0 \\
0 & 0 & 1 \\
0 & 0 & 0
\end{array}\right],\left[\begin{array}{lll}
0 & 0 & 0 \\
0 & 1 & 0 \\
0 & 0 & 1
\end{array}\right],\left[\begin{array}{lll}
0 & 0 & 0 \\
0 & 1 & 1 \\
0 & 0 & 1
\end{array}\right]\right\} .
$$

It is easy to check, that for any pair of matrices $C \in \mathbb{A}$ and $D \in \mathbb{B}, C$ and $D$ do not commute, so that the distance between $A$ and $B$ is at least 4 and thus $\operatorname{diam}\left(\Gamma\left(\mathcal{M}_{3}(\mathcal{B})\right)\right)=$ 4 .

Now, let $n \geq 4$. Let $A, B \in \mathcal{M}_{n}(\mathcal{B})$ be

$$
A=\left[\begin{array}{ccccc}
0 & 0 & \cdots & 0 & 1 \\
0 & & & \\
1 & & & \\
\vdots & & J_{n-1}^{T} & \\
1 & & &
\end{array}\right], \quad B=\left[\begin{array}{cc}
1 & 0 \\
0 & J_{n-1}
\end{array}\right]
$$

Note that the centre of $\mathcal{M}_{n}(\mathcal{B})$ consists only of $0_{n}$ and $I_{n}$. Suppose that the distance between $A$ and $B$ is at most 3 . Then there exist nonscalar matrices $C, D \in \mathcal{M}_{n}(\mathcal{B})$ such that $A-C-D-B$ is a path in $\Gamma\left(\mathcal{M}_{n}(\mathcal{B})\right)$.

Observe that $C$ is not a diagonal matrix, otherwise all of its diagonal entries are equal, since $C$ commutes with $A$. So, $C+I$ is not in the centre and it commutes with $A$ and $D$. Since $C$ and $C+I$ have the same centralizer, we can therefore assume that all the diagonal entries of $C$ are equal to 1 . 
Now, suppose that $D$ is diagonal. Since it commutes with $B$, it has the form

$$
D=\left[\begin{array}{cc}
1 & 0 \\
0 & 0_{n-1}
\end{array}\right] \quad \text { or } \quad D=\left[\begin{array}{cc}
0 & 0 \\
0 & I_{n-1}
\end{array}\right] \text {. }
$$

In both cases, since $C$ and $D$ commute, $C$ has the form

$$
C=\left[\begin{array}{cc}
1 & 0 \\
0 & C_{1}
\end{array}\right]
$$

Since $C$ commutes with $A, C_{1}$ also commutes with $J_{n-1}^{T}$ and the last row of $C_{1}$ is equal to $\left[\begin{array}{llll}0 & \cdots & 0 & 1\end{array}\right]$. So, $C_{1}=I_{n-1}$ and $C$ is in the centre, which is a contradiction. Thus, $D$ is not diagonal and again we can assume that all diagonal entries of $D$ are equal to 1 .

Now, since $D$ and $B$ commute, we have

$$
C=\left[\begin{array}{cccc}
1 & c_{1,2} & \cdots & c_{1, n} \\
c_{2,1} & 1 & \cdots & c_{2, n} \\
\vdots & \vdots & \ddots & \vdots \\
c_{n, 1} & c_{n, 2} & \cdots & 1
\end{array}\right]
$$$$
\text { and } \quad D=\left[\begin{array}{cccccc}
1 & 0 & 0 & \cdots & 0 & 0 \\
0 & 1 & d_{2} & \cdots & d_{n-2} & d_{n-1} \\
0 & 0 & 1 & \ddots & & d_{n-2} \\
\vdots & \vdots & & \ddots & \ddots & \vdots \\
0 & 0 & 0 & \cdots & 1 & d_{2} \\
0 & 0 & 0 & \cdots & 0 & 1
\end{array}\right] \text {, }
$$

where $d_{i} \neq 0$ for some $i \in\{2,3, \ldots, n-1\}$.

Since $A$ and $C$ commute, we have for every $i=2, \ldots, n-1$ that $0=(A C)_{2, i}=(C A)_{2, i}=$ $c_{2, i+1}$ and $0=(A C)_{2, n}=(C A)_{2, n}=c_{2,1}$, so

$$
c_{2, i}=0 \quad \text { for every } \quad i \neq 2 \text {. }
$$

Also, for every $i=2, \ldots, n-1$ we have $(A C)_{1, i}=(C A)_{1, i}$ and thus

$$
c_{n, i}=c_{1, i+1} .
$$

Further, for every $i=3, \ldots, n-1$ we have $c_{1, i}=c_{1, i}+c_{2, i}=(A C)_{3, i}=(C A)_{3, i}=c_{3, i+1}$ and $c_{1, n}=(A C)_{3, n}=(C A)_{3, n}=c_{3,1}$. For $i \geq j \geq 3$ and using

$$
c_{1, i}+c_{j-1, i}=(A C)_{j, i}=(C A)_{j, i}=c_{j, i+1},
$$

we prove by induction that

$$
c_{j, i+1}=c_{1, i}+c_{1, i-1}+\ldots+c_{1, i-j+3} \quad \text { for every } \quad i \geq j \geq 3
$$

and

$$
c_{j, 1}=c_{1, n}+c_{1, n-1}+\ldots+c_{1, n-j+3} \quad \text { for every } j \geq 3 .
$$

Let $3 \leq i<j \leq n-1$. First $c_{j, 3}=(C A)_{j, 2}=(A C)_{j, 2}=c_{1,2}+c_{j-1,2}$ and $c_{j, i}=(C A)_{j, i-1}=$ $(A C)_{j, i-1}=c_{1, i-1}+c_{j-1, i-1}$ and by induction we have

$$
c_{j, i}=c_{1,2}+\ldots+c_{1, i-1}+c_{j-i+2,2} \quad \text { for every } \quad 3 \leq i<j \leq n-1 .
$$

Since $D$ is not diagonal, at least one of $d_{2}, \ldots, d_{n-1}$ is nonzero. Let $k$ be the greatest index, such that $d_{k} \neq 0$, so $d_{k}=1$. Note that the second row of $D$ is therefore equal to $\left[\begin{array}{lllllllll}0 & 1 & d_{2} & \cdots & d_{k-1} & 1 & 0 & \cdots & 0\end{array}\right]$.

Suppose first that $k=n-1$. Since $C$ and $D$ commute, we have $0=(C D)_{2,1}=$ $(D C)_{2,1}=c_{2,1}+d_{2} c_{3,1}+\ldots+d_{n-1} c_{n, 1}=c_{2,1}+d_{2} c_{3,1}+\ldots+d_{n-2} c_{n-1,1}+c_{n, 1}$, thus $c_{n, 1}=0$ and by (5), we have $c_{1,3}+\ldots+c_{1, n}=c_{n, 1}=0$. It follows that $c_{1, i}=0$ for all $i \geq 3$. Furthermore, $0=c_{1, n}=(D C)_{1, n}=(C D)_{1, n}=c_{1,2} d_{n-1}=c_{1,2}$ so $c_{1,2}=0$ and by (4) it follows that $C$ is lower-triangular. 
Suppose now that $k<n-1$. Since $C$ and $D$ commute, we have $0=(C D)_{2,1}=$ $(D C)_{2,1}=c_{2,1}+d_{2} c_{3,1}+\ldots+d_{k} c_{k+1,1}=c_{2,1}+d_{2} c_{3,1}+\ldots+d_{k-1} c_{k, 1}+c_{k+1,1}$, thus $c_{k+1,1}=$ 0 and by (5) it follows that $c_{1, n-k+2}+\ldots+c_{1, n}=0$. Therefore, $c_{1, i}=0$ for every $i \geq n-k+2$. Furthermore, $0=c_{1, n}=(D C)_{1, n}=(C D)_{1, n}=c_{1, n-k+1} d_{k}=c_{1, n-k+1}$. By induction, $c_{1, n-k-i+2}=c_{1, n-k-i+2} d_{k}=(C D)_{1, n-i+1}=(D C)_{1, n-i+1}=c_{1, n-i+1}=0$ for every $i=1, \ldots, n-k$, so that $c_{1, j}=0$ for every $j \geq 2$ and by (4), $C$ is lower-triangular.

Now, we have $c_{j, i}=0$ for every $i>j$ and by applying (3) it follows that

$$
c_{j, i}=c_{j-1, i-1}=\ldots=c_{j-i+2,2}
$$

for every $3 \leq i<j \leq n-1$. Since $C$ is lower-triangular, (2) implies that $c_{n, i}=0$ for $i \geq 2$ and $c_{n, 1}=0$ by (5). Therefore we have for every $i=2, \ldots, n-2$ that $0=c_{n, i+1}=$ $(C A)_{n, i}=(A C)_{n, i}=c_{n-1, i}$, which by (6) implies that $c_{n-i+1,2}=0$, so again by (6), $c_{j, i}=0$ for all $3 \leq i<j \leq n-1$. Together with (1) we have that $C=I_{n}$, a contradiction.

So, we have proved that the distance between $A$ and $B$ cannot be less than 4 and therefore $\operatorname{diam}\left(\Gamma\left(\mathcal{M}_{n}(\mathcal{B})\right)\right)=4$.

Corollary 2.3. If $S$ is a commutative entire antinegative semiring, then $\operatorname{diam}\left(\Gamma\left(\mathcal{M}_{2}(S)\right)\right)=$ $\infty$ and $\operatorname{diam}\left(\Gamma\left(\mathcal{M}_{n}(S)\right)\right) \geq 4$, for $n \geq 3$.

Proof. For a matrix $A \in \mathcal{M}_{n}(S)$, let us denote by $\operatorname{supp}(A) \in \mathcal{M}_{n}(\mathcal{B})$ the unique $(0,1)$ matrix with the property $A_{i, j} \neq 0$ if and only if $(\operatorname{supp}(A))_{i, j} \neq 0$ for all $1 \leq i, j \leq n$.

Since $S$ is a commutative entire antinegative semiring, $A B=B A$ for $A, B \in \mathcal{M}_{n}(S)$ implies that $\operatorname{supp}(A) \operatorname{supp}(B)=\operatorname{supp}(B) \operatorname{supp}(A)$ for $\operatorname{supp}(A), \operatorname{supp}(B) \in \mathcal{M}_{n}(\mathcal{B})$. Thus, it follows that $\operatorname{diam}\left(\Gamma\left(\mathcal{M}_{n}(S)\right)\right) \geq \operatorname{diam}\left(\Gamma\left(\mathcal{M}_{n}(\mathcal{B})\right)\right)$. Now, the statement follows by Theorem 2.2 .

\section{The COMmUting GRAPH OF The FUlL MATRIX SEMIRING OVER $\mathbb{T}$}

The two operations $\oplus$ and $\odot$ in $\mathbb{T}$ naturally induce the matrix addition and multiplication on the semiring $\left(\mathcal{M}_{n}(\mathbb{T}), \oplus, \odot\right)$, namely for $A=\left[a_{i, j}\right], B=\left[b_{i, j}\right] \in \mathcal{M}_{n}(\mathbb{T})$ we have

$$
\begin{aligned}
& (A \oplus B)_{i, j}=a_{i, j} \oplus b_{i, j}, \text { and } \\
& (A \odot B)_{i, j}=a_{i, 1} \odot b_{1, j} \oplus a_{i, 2} \odot b_{2, j} \oplus \ldots \oplus a_{i, n} \odot b_{n, j} .
\end{aligned}
$$

Let $I_{n}$ be the identity matrix in $\mathcal{M}_{n}(\mathbb{T})$, i.e. matrix with zeros on the diagonal and $-\infty$ offdiagonal, and let $E$ be the matrix with all entries equal to $0 \in \mathbb{R}$. For $a \in \mathbb{T}$ and $A=\left[a_{i, j}\right] \in \mathcal{M}_{n}(\mathbb{T})$ we also define the matrix $a A$ in the natural way, i.e. $(a A)_{i, j}=$ $a \odot a_{i j}=a+a_{i j}$.

It was proved in [7, Cor. 11] that $\operatorname{diam}\left(\Gamma\left(\mathcal{M}_{n}(\mathbb{T})\right)\right) \geq 3$ for all $n \geq 3$. Here, we will prove that $\operatorname{diam}\left(\Gamma\left(\mathcal{M}_{n}(\mathbb{T})\right)\right)=4$ for all $n \geq 3$. 
Lemma 3.1. The centralizer $C_{\mathcal{M}_{n}(\mathbb{T})}(E)$ consists of exactly all matrices $A=\left[a_{i, j}\right]$, such that there exists some element $a \in \mathbb{T}$, with the property

$$
\max _{j}\left\{a_{i, j}\right\}=a \text { for all } i=1,2, \ldots, n \text { and } \max _{i}\left\{a_{i, j}\right\}=a \text { for all } j=1,2, \ldots, n \text {. }
$$

Proof. For the matrix $A=\left[a_{i, j}\right]$ we denote $a=\max _{i, j=1, \ldots, n}\left\{a_{i, j}\right\}$. If $A$ commutes with $E$, then $\max \left\{a_{i, 1}, a_{i, 2}, \ldots, a_{i, n}\right\}=a_{i, 1} \oplus a_{i, 2} \oplus \ldots \oplus a_{i, n}=(A \odot E)_{i, 1}=(E \odot A)_{i, 1}=$ $a_{1,1} \oplus a_{2,1} \oplus \ldots \oplus a_{n, 1}=\max \left\{a_{1,1}, a_{2,1}, \ldots, a_{n, 1}\right\}$ for every $i=1, \ldots, n$. So all rows of $A$ have the same maximum $a$, thus $a$ appears in every row of $A$. Similarly, $a$ appears in every column of $A$. Conversely, if $a$ appears in every row and column of $A$, then clearly $E \odot A=a E=A \odot E$.

Theorem 3.2. For the tropical semiring $\mathbb{T}$, we have $\operatorname{diam}\left(\Gamma\left(\mathcal{M}_{2}(\mathbb{T})\right)\right)=\infty$ and

$$
\operatorname{diam}\left(\Gamma\left(\mathcal{M}_{n}(\mathbb{T})\right)\right)=4
$$

for $n \geq 3$.

Proof. By Corollary 2.3 we have that $\operatorname{diam}\left(\Gamma\left(\mathcal{M}_{2}(\mathbb{T})\right)\right)=\infty$ and $\operatorname{diam}\left(\Gamma\left(\mathcal{M}_{n}(\mathbb{T})\right)\right) \geq 4$.

Suppose now $n \geq 3$. If $D=\left[d_{k, l}\right]$ is a diagonal matrix, where $d_{i, i}=d_{j, j}$ for some $i, j$, then let us define the $n \times n$ matrix $F=\left[f_{k, l}\right]$, such that

$$
f_{k, l}= \begin{cases}0, & k=l \text { or }(k, l)=(i, j) \text { or }(k, l)=(j, i), \\ -\infty, & \text { otherwise }\end{cases}
$$

Now, $(F \odot D)_{j, i}=(F \odot D)_{i, j}=d_{j, j}=d_{i, i}=(D \odot F)_{i, j}=(D \odot F)_{j, i}$. Furthermore, $(F \odot D)_{k, k}=d_{k, k}=(D \odot F)_{k, k}$ for all $k$ and $(F \odot D)_{k, l}=-\infty=(D \odot F)_{k, l}$ for all $k \neq l$, and $\{k, l\} \neq\{i, j\}$. Thus $D-F-E$ is a path in $\Gamma\left(\mathcal{M}_{n}(\mathbb{T})\right)$ by Lemma 3.1.

If $A=\left[a_{i, j}\right]$ is a nondiagonal matrix, let $a=\max \left\{a_{i, j}\right\}$, and then, $A-A \oplus a I_{n}-E$ is a path in $\Gamma\left(\mathcal{M}_{n}(\mathbb{T})\right)$ by Lemma 3.1 .

We have thus proved that $d(A, E) \leq 2$ for all matrices $A$ except for the diagonal matrices with all of their diagonal entries distinct. We will now prove that $d(D, A) \leq 4$ for a diagonal matrix $D$ with all diagonal entries distinct and an arbitrary $A \in \mathcal{M}_{n}(\mathbb{T})$.

If $A$ is diagonal as well, it is clear that $d(D, A)=1$. So, suppose $A=\left[a_{i, j}\right]$ is nondiagonal and let $\mu=\max \left\{a_{i, j}\right\}$ and $\varepsilon=\min \left\{a_{i, j} ; a_{i, j} \neq-\infty\right\}$. Denote by $B=\left[b_{i, j}\right]$ the $n \times n$ matrix, defined by

$$
b_{i, j}= \begin{cases}\mu, & i=j \\ \varepsilon, & i \neq j .\end{cases}
$$

Note that $\varepsilon+a_{i, j} \leq \varepsilon+\mu \leq a_{k, l}+\mu$ for all $i, j, k, l$ and thus for every $1 \leq i, j \leq n$ we have $(A \odot B)_{i, j}=a_{i, j}+\mu=(B \odot A)_{i, j}$.

For the matrix

$$
C=\left[\begin{array}{ccc}
\mu & \varepsilon & -\infty \\
\varepsilon & \mu & \\
-\infty & \mu I_{n-2}
\end{array}\right]
$$

it is easy to see that $B \odot C=C \odot B$. Therefore,

$$
D-\left[\begin{array}{cc}
d_{1,1} I_{2} & -\infty \\
-\infty & d_{2,2} I_{n-2}
\end{array}\right]-C-B-A
$$


is a path in $\Gamma\left(\mathcal{M}_{n}(\mathbb{T})\right)$ and thus $d(D, A) \leq 4$.

As a Corollary of [7, Thm. 7] we have the following.

Proposition 3.3. For the tropical semiring $\mathbb{T}$, we have

$$
\operatorname{diam}\left(\Gamma\left(\mathrm{GL}_{n}(\mathbb{T})\right)\right)= \begin{cases}5, & n \text { prime } \\ \infty, & \text { otherwise }\end{cases}
$$

Remark. Note that [2, Thm. 14, 15] state that for an infinite commutative division ring $R$, we have the equality

$$
\operatorname{diam}\left(\Gamma\left(\mathcal{M}_{n}(R)\right)\right)=\operatorname{diam}\left(\Gamma\left(\mathrm{GL}_{n}(R)\right)\right)
$$

for all $n \geq 2$. With Theorem 3.2 and Proposition 3.3, we proved that this result cannot be generalized to commutative division semirings.

\section{The COMmUting GRAPH OF THE FUll MATRIX SEMIRING OVER A NONENTIRE COMMUTATIVE SEMIRING}

In this section, we prove that the diameter of the commuting graph of the full matrix semiring over a nonentire commutative semiring is always equal to 3 . This generalizes the result from [8, Thm. 1.1], where it has been proved that the diameter of the commuting graph of the full matrix ring over the ring $\mathbb{Z}_{m}$ for a composite number $m$ is always equal to 3 .

First, we find the lower bound for the diameter of the commuting graph of matrices over an arbitrary commutative semiring.

Proposition 4.1. If $S$ is a commutative semiring, then $\operatorname{diam}\left(\Gamma\left(\mathcal{M}_{n}(S)\right)\right) \geq 3$ for $n \geq 2$.

Proof. By Lemma 2.1, the only matrices in the intersection of the centralizer of $J_{n}$ and the centralizer of $J_{n}^{T}$ are scalar matrices, but these are of course central. Thus, the distance between matrices $J_{n}$ and $J_{n}^{T}$ in $\Gamma\left(\mathcal{M}_{n}(S)\right)$ is at least 3 .

We now prove that this bound is sharp, since the diameter is equal to 3 for all commuting graphs of matrices over nonentire commutative semirings.

Theorem 4.2. If $S$ is a nonentire commutative semiring, then $\operatorname{diam}\left(\Gamma\left(\mathcal{M}_{n}(S)\right)\right)=3$ for $n \geq 2$.

Proof. Since $S$ is not entire, there exist nonzero $x, y \in S$ such that $x y=y x=0$. (Note that it may happen that $x=y$.) Choose arbitrary noncentral matrices $A, B \in \mathcal{M}_{n}(S)$.

Case 1: Suppose $x A$ and $y B$ are noncentral matrices in $\mathcal{M}_{n}(S)$. Then $A-x A-y B-B$ is a path of length 3 in $\Gamma\left(\mathcal{M}_{n}(S)\right)$. 
Case 2: Suppose $x A$ is central and $y B$ is a noncentral matrix in $\mathcal{M}_{n}(S)$. Then $A x E_{1,2}=$ $x A E_{1,2}=E_{1,2} x A=x E_{1,2} A$, so $A-x E_{1,2}-y B-B$ is a path of length 3 in $\Gamma\left(\mathcal{M}_{n}(S)\right)$. Similarly, we treat the case when $y B$ is central and $x A$ is not.

Case 3: Suppose $x A$ and $y B$ are central matrices in $\mathcal{M}_{n}(S)$. Then $A-x E_{1,2}-y E_{1,2}-B$ is a path of length 3 in $\Gamma\left(\mathcal{M}_{n}(S)\right)$.

The three cases considered show that the distance between any two matrices in $\mathcal{M}_{n}(S)$ is at most 3, and therefore $\operatorname{diam}\left(\Gamma\left(\mathcal{M}_{n}(S)\right)\right) \leq 3$. By Proposition 4.1, it follows that $\operatorname{diam}\left(\Gamma\left(\mathcal{M}_{n}(S)\right)\right)=3$.

\section{REFERENCES}

[1] A. Abdollahi, Commuting graphs of full matrix rings over finite fields, Linear Algebra Appl., 428 (2008), 2947-2954.

[2] S. Akbari, A. Mohammadian, H. Radjavi, P. Raja: On the diameters of commuting graphs, Linear Algebra Appl., 418 (2006), 161-176.

[3] S. Akbari, P. Raja: Commuting graphs of some subsets in simple rings, Linear Algebra Appl., 416 (2006), 1038-1047.

[4] M. Akian, S. Gaubert, A. Guterman: Linear independence over tropical semirings and beyond, Tropical and idempotent mathematics, 138, Contemp. Math., 495, Amer. Math. Soc., Providence, RI, 2009.

[5] M. Develin, F. Santos, B. Sturmfels: On the rank of a tropical matrix, Combinatorial and computational geometry, Math. Sci. Res. Inst. Publ., 52, Cambridge Univ. Press, Cambridge, 2005, 213-242.

[6] G. Dolinar, B. Kuzma, P. Oblak: On maximal distances in a commuting graph, preprint.

[7] D. Dolžan, P. Oblak: Commuting graphs of matrices over semirings, Linear Algebra Appl., doi: 10.1016/j.laa.2010.04.014.

[8] M. Giudici, A. Pope: The diameters of commuting graphs of linear groups and matrix rings over the integers modulo m, Australasian Journal of Combinatorics, 48 (2010), 221-230.

[9] A. Iranmanesh, A. Jafarzadeh: On commuting graph associated with the symmetric and alternating groups, J. Alg. Appl., 7 (2008), no. 1, 129-146.

[10] R. D. Katz, H. Schneider, S. Sergeev: On commuting matrices in max algebra and in classical nonnegative algebra, Linear Algebra Appl., doi: 10.1016/j.laa.2010.08.027.

[11] A. Mohammadian, On commuting graphs of finite matrix rings, Commun. Algebra, 38 (2010), 988-994.

[12] Y. Segev: The commuting graph of minimal nonsolvable groups, Geom. Dedicata, 88 (2001), $55-66$.

[13] I. Simon: On semigroups of matrices over the tropical semiring, RAIRO Inform. Thor. Appl., 28 (1994), no. 3-4, 277-294.

D. Dolžan: Department of Mathematics, Faculty of Mathematics and Physics, University of Ljubljana, Jadranska 19, SI-1000 LjublJana, Slovenia; E-Mail: David.dolzan@FmF.Uni-LJ.SI

D. Kokol Bukovšek: Department of Mathematics, Faculty of Mathematics and Physics,

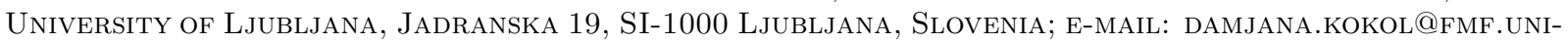
LJ.SI

P. Oblak: Faculty of Computer and Information Science, Tržaška 25, SI-1000 Ljubluana, SLOVENIA; E-MAIL: POLONA.OBLAK@FRI.UNI-LJ.SI 